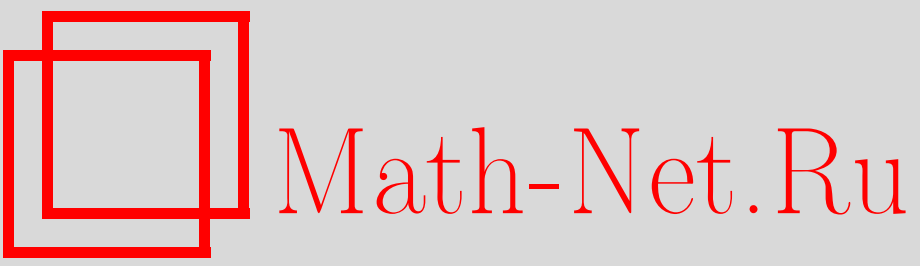

А. М. Переломов, Волчок Ковалевской. Элементарный подход, ТМФ, 2002, том 131, номер 2, 197-205

DOI: https://doi.org/10.4213/tmf324

Использование Общероссийского математического портала Math-Net.Ru подразумевает, что вы прочитали и согласны с пользовательским соглашением

http://www.mathnet.ru/rus/agreement

Параметры загрузки:

IP : 3.80 .181 .102

26 апреля 2023 г., 13:51:07 


\section{ВОЛЧОК КОВАЛЕВСКОЙ. ЭЛЕМЕНТАРНЫЙ ПОДХОД}

Цель данной заметки - дать элементарное и краткое решение уравнений движения волчка Ковалевской. Для этого мы используем некоторые результаты оригинальных работ Ковалевской, Шёттера и Вебера, а также принадлежащее автору представление Лакса.

\section{Памяти Юргена Мозера}

1. Волчок Ковалевской [1] является одним из замечательных примеров интегрируемых систем. Это волчок, главные моменты инерции которого $J_{1}, J_{2}, J_{3}$ удовлетворяют соотношениям

$$
J_{1}=J_{2}=2 J_{3}=J
$$

а центр масс лежит в экваториальной плоскости тела (для простоты мы в дальнейшем полагаем $J=1)$. Динамическими переменными являются компоненты $m_{1}, m_{2}, m_{3}$ момента импульса и компоненты $n_{1}, n_{2}, n_{3}$ вектора центра масс в системе, связанной с главными осями тела.

Данная система является гамильтоновой относительно пуассоновой структуры, связанной с алгеброй Ли $e(3)$ движений трехмерного евклидова пространства,

$$
\left\{m_{i}, m_{j}\right\}=\varepsilon_{i j k} m_{k}, \quad\left\{m_{i}, n_{j}\right\}=\varepsilon_{i j k} n_{k}, \quad\left\{n_{i}, n_{j}\right\}=0,
$$

где $\varepsilon_{i j k}$ - стандартный полностью кососимметричный тензор.

Гамильтониан имеет вид

$$
H=\frac{1}{2}\left(m_{1}^{2}+m_{2}^{2}+2 m_{3}^{2}-n_{1}\right)
$$

и уравнения движения выглядят как (точка означает производную по времени)

$$
\dot{m}_{j}=\left\{H, m_{j}\right\}, \quad \dot{n}_{j}=\left\{H, n_{j}\right\}
$$

* Институт теоретической и экспериментальной физики, Москва, Россия; Departamento de Física, Facultad de Ciencias, Universidad de Oviedo, Oviedo, Spain.

E-mail: perelomo@dftuz.unizar.es 
или в явном виде

$$
\begin{gathered}
\dot{m}_{1}=m_{2} m_{3}, \quad 2 \dot{m}_{2}=-\left(2 m_{3} m_{1}+n_{3}\right), \quad 2 \dot{m}_{3}=n_{2}, \\
\dot{n}_{1}=2 m_{3} n_{2}-m_{2} n_{3}, \quad \dot{n}_{2}=m_{1} n_{3}-2 m_{3} n_{1}, \quad \dot{n}_{3}=m_{2} n_{1}-m_{1} n_{2} .
\end{gathered}
$$

Заметим, что вектор угловой скорости имеет вид

$$
(p, q, r)=\left(m_{1}, m_{2}, 2 m_{3}\right) .
$$

В знаменитой работе [1] Ковалевской удалось проинтегрировать эти уравнения в терминах абелевых функций двух переменных. Подход Ковалевской был позднее упрощен Шёттером [2]. Отметим также работу Колосова [3], в которой эта задача была сведена к задаче о движении точки на плоскости в потенциальном поле.

Прошло столетие, и волчок Ковалевской снова привлек к себе интерес. В работе [4] он был рассмотрен как проекция волчка Эйлера. Этот подход дает как объяснение знаменитому соотношению (1), так и естественное многомерное интегрируемое обобшение такой системы.

В работах [5], [6] были найдены нетривиальные редукции, дающие эллиптические решения для волчка Ковалевской. В работе [7] (см. также [8] и приведенную там библиографию) были построены переменные действие-угол для этой задачи и обнаружена пуассонова коммутативность переменных $s_{1}$ и $s_{2}$. Заметим, что именно эти переменные возникают при рассмотрении волчка Ковалевской как проекции волчка Эйлера.

Авторы ряда работ (см. [9]-[12]) использовали алгебро-геометрический подход к данной задаче. К сожалению, этот подход очень сложен и к настояшему времени в его рамках воспроизведена только некоторая часть исходных результатов для волчка Ковалевской. Например, представление Лакса со спектральным параметром [9], [11] дает спектральную кривую рода три и соответственно абелевы функции трех переменных, а не двух, как в оригинальной работе Ковалевской [1]. Даже в простейшем случае $(m, n)=0$ провести аналогию между двумя такими подходами весьма сложно [12]. Поэтому, по мнению автора, элементарный и естественный подход Ковалевской-Шёттера остается более адекватным для рассматриваемой задачи.

2. Следуя [1] и [2], сначала напомним, что уравнения (5) имеют четыре интеграла движения:

$$
\begin{aligned}
H_{1} & =2 H=m_{1}^{2}+m_{2}^{2}+2 m_{3}^{2}-n_{1}=h_{1}, \\
\widetilde{H}_{2} & =\xi_{+} \xi_{-}=k^{2}, \\
C_{3} & =(m, n)=m_{1} n_{1}+m_{2} n_{2}+m_{3} n_{3}=c_{3}, \\
C_{4} & =n_{1}^{2}+n_{2}^{2}+n_{3}^{2}=c_{4},
\end{aligned}
$$

где

$$
\xi_{ \pm}=m_{ \pm}^{2}+n_{ \pm}, \quad m_{ \pm}=\left(m_{1} \pm i m_{2}\right), \quad n_{ \pm}=\left(n_{1} \pm i n_{2}\right) .
$$

Заметим, что $C_{3}$ и $C_{4}$ являются функциями Казимира, а уравнения (9), (10) определяют четырехмерное симплектическое многообразие $\mathcal{M}_{c}$ - орбиту коприсоединенного представления группы Ли $E(3)$ (группы движений трехмерного евклидова пространства).

Интегрирование уравнений (5) состоит из нескольких шагов. 
3. Начнем с представления Лакса [4], описывающего волчок Ковалевской как проекцию волчка Эйлера,

$$
\begin{aligned}
\dot{L}_{2} & =\left[L_{2}, M_{2}\right], \\
L_{2} & =-A\left(2 \widehat{m}^{2}+(\gamma \otimes n+n \otimes \gamma)\right) A, \\
M_{2} & =-A \widehat{m} A,
\end{aligned}
$$

где

$$
\begin{gathered}
\widehat{m}=\left(\begin{array}{ccc}
0 & m_{3} & -m_{2} \\
-m_{3} & 0 & m_{1} \\
m_{2} & -m_{1} & 0
\end{array}\right), \quad A=\left(\begin{array}{ccc}
1 & 0 & 0 \\
0 & 1 & 0 \\
0 & 0 & 0
\end{array}\right), \quad \gamma=(1,0,0), \quad n=\left(n_{1}, n_{2}, n_{3}\right), \\
\operatorname{tr} L_{2}=2 H_{1}=4 H, \quad \operatorname{det} L_{2}=H_{2}=H_{1}^{2}-\widetilde{H}_{2}
\end{gathered}
$$

Тогда

$$
\begin{gathered}
\operatorname{det}\left(s I-L_{2}\right)=s P_{2}(s), \quad P_{2}(s)=s^{2}-2 H_{1} s+H_{2}, \\
H_{1}=h_{1}, \quad H_{2}=h_{2}, \quad h_{2}=h_{1}^{2}-k^{2} .
\end{gathered}
$$

Для волчка Ковалевской уравнения движения содержат как квадратичные, так и линейные члены по динамическим переменным $m_{1}, m_{2}, m_{3}, n_{1}, n_{2}, n_{3}$. Отсюда следует, что некоторые из этих переменных, являясь мероморфными функциями времени $t$, имеют полюсы второго порядка по $t$.

Постараемся найти такую замену переменных, чтобы уравнения движения содержали только квадратичные члены. Этого можно достичь исключением переменных $n_{1}$ и $n_{2}$.

Из уравнений (7)-(10) следуют два линейных уравнения на переменные $n_{1}$ и $n_{2}$

$$
\begin{aligned}
m_{1} n_{1}+m_{2} n_{2} & =c_{3}-m_{3} n_{3}, \\
\left(m_{1}^{2}-m_{2}^{2}\right) n_{1}+2 m_{1} m_{2} n_{2} & =\frac{1}{2}\left(n_{3}^{2}-\left(m_{1}^{2}+m_{2}^{2}\right)^{2}+k^{2}-c_{4}\right) .
\end{aligned}
$$

Используя их, мы можем исключить $n_{1}$ и $n_{2}$ из уравнений

$$
2 H_{1}=2 h_{1}, \quad H_{2}=h_{2} .
$$

При этом обнаруживается, что левые части этих уравнений становятся квадратичными формами, если ввести новые переменные ${ }^{1)} f=\left(f_{1}, f_{2}, f_{3}\right), g=\left(g_{1}, g_{2}, g_{3}\right)$, где

$$
\begin{aligned}
& f_{1}=\frac{1}{m_{2}}, \quad f_{2}=\frac{m_{1}}{m_{2}}, \quad f_{3}=\frac{m_{1}^{2}+m_{2}^{2}}{m_{2}}, \\
& g_{1}=2 \frac{m_{3}}{m_{2}}, \quad g_{2}=\frac{n_{3}}{m_{2}}, \quad g_{3}=-\frac{2}{m_{2}}\left(\left(m_{1}^{2}+m_{2}^{2}\right) m_{3}+m_{1} n_{3}\right) \text {. }
\end{aligned}
$$

Тогда будем иметь

$$
\begin{aligned}
2 H_{1} & =S_{1}(f)+T_{1}(g), \\
H_{2} & =S_{2}(f)+T_{2}(g),
\end{aligned}
$$

\footnotetext{
1) Заметим, что эти переменные уже использовались в работах [1] и [2].
} 
где

$$
\begin{aligned}
& S_{1}=\frac{1}{2}\left(\left(f_{3}+h_{1} f_{1}\right)^{2}-4 c_{3} f_{1} f_{2}-\left(c_{4}+h_{2}\right) f_{1}^{2}-4 h_{1} f_{2}^{2}\right) \\
& S_{2}=-2 c_{3}\left(f_{3}+h_{1} f_{1}\right) f_{2}-\left(c_{4}+h_{2}\right) f_{2}^{2}-c_{3}^{2} f_{1}^{2} \\
& T_{1}=\frac{1}{2}\left(-g_{1} g_{3}+g_{2}^{2}\right) \\
& T_{2}=\frac{1}{4}\left(\left(h_{1} g_{1}-g_{3}\right)^{2}-\left(c_{4}+h_{2}\right) g_{1}^{2}+4 c_{3} g_{1} g_{2}\right) .
\end{aligned}
$$

Из (20) следует, что величины $S_{1}(f)$ и $S_{2}(f)$ можно рассматривать как естественную проекцию двух нетривиальных интегралов движения $2 \mathrm{H}_{1}$ и $\mathrm{H}_{2}$,

$$
\pi: 2 H_{1} \rightarrow S_{1}(f), \quad \pi: H_{2} \rightarrow S_{2}(f)
$$

Поэтому примем их за новые динамические переменные. Естественно также записать (аналогично (15)) выражение

$$
\mathcal{F}(s, f)=s^{2}-S_{1}(f) s+S_{2}(f)
$$

и рассмотреть уравнение

$$
\mathcal{F}(s, f)=0 .
$$

Корни $s_{1}$ и $s_{2}$ уравнения (27) являются знаменитыми переменными Ковалевской

$$
s_{1}+s_{2}=S_{1}, \quad s_{1} s_{2}=S_{2} .
$$

Как было показано в работе [7], переменные $s_{1}$ и $s_{2}$ коммутируют относительно скобки Пуассона,

$$
\left\{s_{1}, s_{2}\right\}=0, \quad\left\{S_{1}, S_{2}\right\}=0 .
$$

Другое свойство этих переменных можно записать как

$$
\left\{T_{1}, T_{2}\right\}=0, \quad 2\left\{H_{1}, S_{2}\right\}=\left\{H_{2}, S_{1}\right\} .
$$

Таким образом, имеется также однопараметрическое семейство переменных $S_{1}(\lambda)=$ $S_{1}+2 \lambda H_{1}$ и $S_{2}(\lambda)=S_{2}+\lambda H_{2}$, коммутирующих относительно скобки Пуассона,

$$
\left\{S_{1}(\lambda), S_{2}(\lambda)\right\}=0
$$

Заметим, что функции $f_{j}, g_{k}$ не являются независимыми, но удовлетворяют соотношениям

$$
\begin{gathered}
f_{1} f_{3}-f_{2}^{2}=1, \\
f_{1} g_{3}+2 f_{2} g_{2}+f_{3} g_{1}=0 .
\end{gathered}
$$

Эти соотношения являются стандартными для кокасательного расслоения двумерного двулистного гиперболоида. Итак, после замены переменных мы получили динамическую систему на двумерном двулистном гиперболоиде. 
В новых переменных уравнения движения (5) принимают вид

$$
\begin{aligned}
& \dot{f}_{1}=\frac{1}{2}\left(f_{1} g_{2}+f_{2} g_{1}\right), \\
& \dot{f}_{2}=-\frac{1}{2}\left(f_{1} g_{3}+f_{2} g_{2}\right)=\frac{1}{4}\left(-f_{1} g_{3}+f_{3} g_{1}\right), \\
& \dot{f}_{3}=-\frac{1}{2}\left(f_{2} g_{3}+f_{3} g_{2}\right)
\end{aligned}
$$

$$
\begin{aligned}
& \dot{g}_{1}=c_{3} f_{1}^{2}+h_{1} f_{1} f_{2}-f_{2} f_{3}, \\
& \dot{g}_{2}=\frac{1}{2} \gamma f_{1}^{2}+c_{3} f_{1} f_{2}+\frac{1}{2} f_{3}^{2}, \\
& \dot{g}_{3}=-c_{3}\left(f_{1} f_{3}+2 f_{2}^{2}\right)-h_{1} f_{2} f_{3}-\gamma_{4} f_{1} f_{2},
\end{aligned}
$$

где

$$
\gamma_{4}=c_{4}-k^{2}=c_{4}+h_{2}-h_{1}^{2} .
$$

Отметим также следующие полезные уравнения:

$$
\begin{aligned}
\ddot{f}_{1} & =\nu f_{1}+\frac{1}{2}\left(h_{1} f_{1}+f_{3}\right), \\
\ddot{f}_{2} & =\nu f_{2}+\left(h_{1} f_{2}+\frac{1}{2} c_{3} f_{1}\right), \\
\ddot{f_{3}} & =\nu f_{3}+\frac{1}{2}\left(h_{1} f_{3}-2 c_{3} f_{2}-\left(c_{4}-k^{2}\right) f_{1}\right), \\
\nu & =h_{1}-S_{1} .
\end{aligned}
$$

Можно показать, что уравнения движения для $f_{1}, f_{2}, f_{3}$ имеют лаксов вид

$$
\dot{L}=[L, M],
$$

где

$$
L=\left(\begin{array}{cc}
f_{2} & f_{1} \\
-f_{3} & -f_{2}
\end{array}\right), \quad M=\frac{1}{4}\left(\begin{array}{ll}
-g_{2} & g_{1} \\
-g_{3} & g_{2}
\end{array}\right) .
$$

Уравнения для $g_{1}, g_{2}, g_{3}$ имеют вид

$$
\dot{M}=[L, N],
$$

где

$$
N=-\frac{1}{8}\left(\begin{array}{cc}
c_{3} f_{1}+2 h_{1} f_{2} & f_{3}+h_{1} f_{1} \\
\gamma_{4} f_{1}+2 c_{3} f_{2}-h_{1} f_{3} & -\left(c_{3} f_{1}+2 h_{1} f_{2}\right)
\end{array}\right) .
$$

Рассмотрим теперь задачу Клебша [13] (см. также [14]-[17]), т.е. задачу о движении твердого тела в идеальной жидкости. Динамическими переменными здесь являются компоненты импульса $p_{1}, p_{2}, p_{3}$ и момента импульса $l_{1}, l_{2}, l_{3}$, а в специальном случае они удовлетворяют также дополнительному условию $(l, p)=0$. Эта система является 
гамильтоновой по отношению к пуассоновой структуре для алгебры Ли $e(3)$ движений трехмерного евклидова пространства

$$
\left\{l_{i}, l_{j}\right\}=\varepsilon_{i j k} l_{k}, \quad\left\{l_{i}, p_{j}\right\}=\varepsilon_{i j k} p_{k}, \quad\left\{p_{i}, p_{j}\right\}=0,
$$

где $\varepsilon_{i j k}$ - стандартньй полностью кососимметричньй тензор.

Гамильтониан имеет вид

$$
H=\frac{1}{2}\left(\sum_{j=1}^{3} l_{j}^{2}+\sum_{j, k=1}^{3} B_{j k} p_{j} p_{k}\right),
$$

где величины $B_{j k}$ - константы. Можно проверить, что уравнения движения в этом случае имеют тот же вид, что и уравнения (33), (34), и что второе уравнение в (32) эквивалентно условию $(l, p)=0$.

Заметим, что для диагональной матрицы $B$ эта задача была решена Вебером [18] в терминах абелевых функций двух переменных.

4. Последний шаг состоит в сведении нашей задачи к случаю диагональной матрицы $B$. Для этого удобно использовать важное тождество, открытое Шёттером [2]:

$$
-2 s \mathcal{F}(s)=Q_{2}^{2}(s)-P_{3}(s) Q_{1}(s),
$$

где

$$
\begin{aligned}
& P_{3}(s)=s P_{2}(s)+c_{4} s-2 c_{3}^{2}, \\
& P_{2}(s)=s^{2}-2 h_{1} s+h_{2}, \quad P_{5}(s)=P_{3}(s) P_{2}(s), \\
& Q_{1}(s)=f_{1}^{2} s-2 f_{2}^{2}, \\
& Q_{2}(s)=s^{2} f_{1}-\left(f_{3}+h_{1} f_{1}\right) s-2 c_{3} f_{2} .
\end{aligned}
$$

Вместо переменных $f_{j}, g_{k}$ введем переменные $x_{j}, y_{k}$ по формулам ${ }^{2)}$

$$
\begin{aligned}
& x_{j}=\sqrt{\left(s_{1}-a_{j}\right)\left(s_{2}-a_{j}\right)}, \\
& y_{j}=\frac{x_{k} x_{l}}{s_{1}-s_{2}}\left(\frac{\sqrt{P_{5}\left(s_{1}\right)}}{\left(s_{1}-a_{k}\right)\left(s_{1}-a_{l}\right)}-\frac{\sqrt{P_{5}\left(s_{2}\right)}}{\left(s_{2}-a_{k}\right)\left(s_{2}-a_{l}\right)}\right),
\end{aligned}
$$

где $a_{j}$ - корень уравнения $P_{3}(s)=0$ и $\{j, k, l\}$ - циклическая перестановка множества $\{1,2,3\}$.

Из (45)-(48) получаем выражения для $f_{j}$ через $x_{k}$

$$
\begin{aligned}
& f_{1}=-i \sum_{j=1}^{3} \frac{\sqrt{2 a_{j}}}{P_{3}^{\prime}\left(a_{j}\right)} x_{j} \\
& f_{2}=i \sum_{j=1}^{3} \frac{\sqrt{a_{k} a_{l}}}{P_{3}^{\prime}\left(a_{j}\right)} x_{j} \\
& f_{3}=-h_{1} f_{1}-2 i \sum_{j=1}^{3} \frac{\sqrt{2 a_{j}}}{P_{3}^{\prime}\left(a_{j}\right)}\left(a_{1}+a_{2}+a_{3}-a_{j}\right) x_{j}
\end{aligned}
$$

\footnotetext{
2) Формулы такого типа, введенные Вейерштрассом, очень полезны в теории абелевых функций.
} 


$$
\begin{aligned}
& g_{1}=-i \sum_{j=1}^{3} \frac{\sqrt{2 a_{j}}}{P_{3}^{\prime}\left(a_{j}\right)} y_{j}, \\
& g_{2}=i \sum_{j=1}^{3} \frac{\sqrt{a_{k} a_{l}}}{P_{3}^{\prime}\left(a_{j}\right)} y_{j}, \\
& g_{3}=-h_{1} g_{1}-2 i \sum_{j=1}^{3} \frac{\sqrt{2 a_{j}}}{P_{3}^{\prime}\left(a_{j}\right)}\left(a_{1}+a_{2}+a_{3}-a_{j}\right) y_{j} .
\end{aligned}
$$

Заметим также, что

$$
Q_{2}\left(a_{j}\right)=i \sqrt{2 a_{j}} x_{j}, \quad j=1,2,3 .
$$

Легко видеть, что переменные $x_{j}, y_{k}$ удовлетворяют уравнениям движения для специального случая Клебша $((l, p)=0)$ с диагональной матрицей $B$. Таким образом, можно использовать решение Вебера [18], задаваемое формулами

$$
x_{j}=x_{j 0} \frac{\theta_{j 4}\left(u_{1}, u_{2}\right)}{\theta_{0}\left(u_{1}, u_{2}\right)}, \quad y_{j}=y_{j 0} \frac{\theta_{j}\left(u_{1}, u_{2}\right)}{\theta_{0}\left(u_{1}, u_{2}\right)}, \quad j=1,2,3,
$$

где $x_{j 0}$ и $y_{j 0}$ - константы, $u_{1}$ и $u_{2}$ - линейные функции $t$, а $\theta_{0}\left(u_{1}, u_{2}\right), \theta_{j}\left(u_{1}, u_{2}\right)$ и $\theta_{j 4}\left(u_{1}, u_{2}\right)$ - стандартные тэта-функции с полуцелыми тэта-характеристиками

$$
\begin{aligned}
& \theta_{1}=\theta\left[\begin{array}{ll}
1 & 0 \\
1 & 1
\end{array}\right]\left(u_{1}, u_{2}\right), \quad \theta_{2}=\theta\left[\begin{array}{ll}
0 & 1 \\
0 & 1
\end{array}\right]\left(u_{1}, u_{2}\right), \quad \theta_{3}=\theta\left[\begin{array}{ll}
1 & 1 \\
1 & 0
\end{array}\right]\left(u_{1}, u_{2}\right), \\
& \theta_{14}=\theta\left[\begin{array}{ll}
0 & 0 \\
0 & 1
\end{array}\right]\left(u_{1}, u_{2}\right), \quad \theta_{24}=\theta\left[\begin{array}{ll}
1 & 1 \\
1 & 1
\end{array}\right]\left(u_{1}, u_{2}\right), \quad \theta_{34}=\theta\left[\begin{array}{ll}
0 & 1 \\
0 & 0
\end{array}\right]\left(u_{1}, u_{2}\right), \\
& \theta_{0}=\theta\left[\begin{array}{ll}
0 & 0 \\
0 & 0
\end{array}\right]\left(u_{1}, u_{2}\right) \text {. }
\end{aligned}
$$

Эти функции определяются стандартными формулами

$$
\begin{aligned}
& \theta\left[\begin{array}{ll}
\varepsilon_{1} & \varepsilon_{2} \\
\delta_{1} & \delta_{2}
\end{array}\right]\left(u_{1}, u_{2}\right)= \\
& \quad=\sum_{n_{j}, n_{k}=-\infty}^{\infty} \exp \left\{i \pi\left[\tau_{j k}\left(n_{j}+\frac{\varepsilon_{j}}{2}\right)\left(n_{k}+\frac{\varepsilon_{k}}{2}\right)+\left(n_{j}+\frac{\varepsilon_{j}}{2}\right)\left(2 u_{j}+\delta_{j}\right)\right]\right\},
\end{aligned}
$$

где $\tau_{j k}$-матрица периодов, связанная с алгебраической кривой

$$
y^{2}=P_{5}(x)
$$

Таким образом, формулы (18), (19), (49)-(54) и (56)-(59) дают явное решение для волчка Ковалевской (подробности см. в [1], [2], [18]). 
5. Аналогично подходу Вейерштрасса для геодезических на эллипсоиде [19] мы можем получить еще одно важное тождество,

$$
\mathcal{F}(s) \mathcal{H}(s)-\mathcal{G}^{2}(s)=2 P_{5}(s)
$$

где

$$
\begin{aligned}
\mathcal{F}(s) & =s^{2}-S_{1} s+S_{2} \\
\mathcal{G}(s) & =\dot{S}_{1} s-\dot{S}_{2} \\
\mathcal{H}(s) & =2\left(s^{3}-b_{1} s^{2}+b_{2} s-b_{3}\right), \\
P_{5}(s) & =\left(s^{3}-2 h_{1} s^{2}+\left(c_{4}+h_{2}\right) s-2 c_{3}^{2}\right)\left(s^{2}-2 h_{1} s+h_{2}\right)
\end{aligned}
$$

$$
\begin{aligned}
b_{1}= & -S_{1}+4 h_{1}, \\
b_{2}= & S_{1}^{2}-S_{2}-4 h_{1} S_{1}+2 h_{2}+4 h_{1}^{2}+c_{4}, \\
b_{3}= & -S_{1}^{3}+4 h_{1} S_{1}^{2}+2 S_{1} S_{2}-4 h_{1} S_{2}-\left(2 h_{2}+4 h_{1}^{2}+c_{4}\right) S_{1}- \\
& \quad-\frac{1}{2} \dot{S}_{1}^{2}+4 h_{1} h_{2}+2 h_{1} c_{4}+2 c_{3}^{2} .
\end{aligned}
$$

Из тождества (60) легко получить уравнения движения в стандартной форме АбеляЯкоби

$$
\dot{s}_{1}=i\left(s_{1}-s_{2}\right)^{-1} \sqrt{2 P_{5}\left(s_{1}\right)}, \quad \dot{s}_{2}=i\left(s_{2}-s_{1}\right)^{-1} \sqrt{2 P_{5}\left(s_{2}\right)},
$$

а также представление Лакса со спектральным параметром $s$ в терминах $(2 \times 2)$-матриц:

$$
\begin{aligned}
\dot{\mathcal{L}}(s) & =[\mathcal{L}(s), \mathcal{M}(s)] \\
\mathcal{L}(s) & =\left(\begin{array}{cc}
\mathcal{G} & \mathcal{F} \\
-\mathcal{H} & -\mathcal{G}
\end{array}\right) \\
\mathcal{M}(s) & =\mathcal{F}^{-1}(s)\left(\begin{array}{ll}
0 & 0 \\
\mathcal{C} & \mathcal{D}
\end{array}\right),
\end{aligned}
$$

где

$$
\mathcal{C}=\left(s-2 T_{1}\right) \mathcal{F}(s)-\frac{1}{2} \mathcal{H}(s), \quad \mathcal{D}=-\mathcal{G}(s)
$$

6. В заключение данной статьи я хотел бы высказать предположение, что подобные результаты верны и для $n$-мерного обобшения волчка Ковалевской, приведенного в работе [4].

Основной результат этой заметки об эквивалентности системы Ковалевской и специального случая системы Клебша был получен в 1983 г. в ходе подготовки обзора о движении твердого тела вокруг закрепленной точки [16]. Позднее я имел возможность обсуждать задачу Ковалевской и другие задачи классической механики с профессором Ю. Мозером. Эти обсуждения оказали огромное влияние на мою точку зрения на задачи классической механики в целом. В частности, профессор Мозер подчеркивал важную роль факторизации полинома Ковалевской $P_{5}(x)$ на полиномы $P_{3}(x)$ и $P_{2}(x) . \mathrm{K}$ сожалению, простого объяснения этого факта нет до сих пор. 
Благодарности. Я глубоко признателен профессору Ю. Мозеру за плодотворные обсуждения. Я благодарен также В. З. Енольскому, А. П. Веселову и рецензенту за их замечания. Наконец, я хотел бы поблагодарить Физический факультет Университета Овьедо за гостеприимство.

\section{Список литературы}

[1] S. Kovalevskaya. Acta Math. 1889. V. 12. P. 177-232.

[2] F. Kötter. Acta Math. 1893. V. 17. P. 209-263.

[3] G. V. Kolosov. Math. Ann. 1902. V. 56. P. 265-272.

[4] A. M. Perelomov. Commun. Math. Phys. 1981. V. 81. P. 239-241.

[5] V. Z. Enolsky. Phys. Lett. A. 1984. V. 100. P. 463-466.

[6] В. З. Энольский. ДАН СССР. 1984. Т. 278. № 2. С. 305-308.

[7] С. П. Новиков, А. П. Веселов. Тр. МИАН. 1984. Т. 165. С. 49-61.

[8] H. Dullin, P. Richter, A. P. Veselov. Regular Chaot. Dynam. 1998. V. 3. P. 18-31.

[9] A. G. Reyman, M. A. Semenov-Tian-Shansky. Lett. Math. Phys. 1987. V. 14. P. 55-61.

[10] M. Adler, P. van Moerbeke. Commun. Math. Phys. 1988. V. 113. P. 659-700.

[11] A. I. Bobenko, A. G. Reyman, M. A. Semenov-Tian-Shansky. Commun. Math. Phys. 1989. V. 122. P. 321-354.

[12] D. G. Markushevich. J. Phys. A. 2001. V. 34. P. 2125-2135.

[13] A. Clebsch. Math. Ann. 1871. V. 3. P. 238-262.

[14] Jü. Moser. Geometry of quadrics and spectral theory. In: Differential Geometry. Proc. of the International Chern Symposium, Berkeley, 1979. Eds. W. Y. Hsiang et al. New York: Springer, 1980. P. $147-188$.

[15] А. М. Переломов. Функц. анализ и его прилож. 1981. Т. 15. № 2. С. 83-85.

[16] A. M. Perelomov. Integrable systems of classical mechanics and Lie algebras. Motion of rigid body around fixed point. Preprint ITEP-147, 1983.

[17] A. M. Perelomov. Regular Chaot. Dynam. 2000. V. 5. P. 89-91.

[18] H. Weber. Math. Ann. 1879. V. 14. P. 173-206.

[19] K. Weierstrass. Monatsberichte Akad. Wiss. zu Berlin. 1861. P. 986.

Поступила в редакцию 12.Х.2001 г., после доработки 16.XI.2001 г. 